Rev Inv Vet Perú 2002; 13 (2): 10-17

\title{
EFECTO INHIBITORIO DE LA PROGESTERONA SOBRE EL DESARROLLO DE LA ONDA FOLICULAR EN LLAMAS
}

\author{
Alexei Santiani A. ${ }^{1}$, Víctor Leyva V. $^{2}$ y Wilber García V. ${ }^{3}$
}

\section{Abstract}

The effect of the exogenous progesterone $\left(\mathrm{P}_{4}\right)$ on the development of follicular waves in llamas was studied in twenty-five females. According to dose and regimen for $P_{4}$ administration, animals were allocated to 5 groups ( $n=5$ ): $\mathrm{G} 0$ (control group), $\mathrm{Gl}$ (30 mg $\mathrm{P}_{4} /$ day for 2 days); $\mathrm{G} 2$ (30 $\mathrm{mg} \mathrm{P}_{4} /$ day for 4 days); $\mathrm{G} 3$ (50 $\mathrm{mg} \mathrm{P}_{4}$ /day for 2 days) and G4 (50 $\mathrm{mg} \mathrm{P}_{4} /$ day for 4 days). Development of dominant $(\geq 7 \mathrm{~mm})$ and subordinated follicles $(>3$ $\mathrm{mm}$ and $<7 \mathrm{~mm}$ ) were monitored daily by transrectal ultrasonography. The minimun size reached by the dominant follicle in groups $\mathrm{G} 1(4.6 \pm 0.9 \mathrm{~mm}), \mathrm{G} 3(5.1 \pm 1.5 \mathrm{~mm})$ and $\mathrm{G} 4(5.3$ $\pm 1.6 \mathrm{~mm}$ ) were smaller $(\mathrm{P}<0.05)$ than group $\mathrm{G} 0(8.5 \pm 1.0 \mathrm{~mm})$. The whole follicular regression of the dominant follicle in groups G1 $(5.5 \pm 2.0 \mathrm{~mm}), \mathrm{G} 2(5.9 \pm 1.4 \mathrm{~mm})$ and G3 $(5.3 \pm 3.3 \mathrm{~mm})$ were larger $(\mathrm{P}<0.05)$ than group $\mathrm{G} 0(0.9 \pm 1.4 \mathrm{~mm})$. The number of subordinated follicles had a significative increase $(P<0.05)$ in groups $G 1$ and $G 4$, on days 2 and 3 in relation to days 7 and 8 post-treatment. Results demonstrate that exogenous progesterone inhibits the development of the dominant and subordinate follicles in llamas during treatment days; however, immediately after the end of the treatment, the number of subordinate follicles increases, due to the regression of the dominant follicle in order to allow the emergence of a new follicular wave.

Key words: progesterone, follicular waves, follicles, llamas

\section{Resumen}

El efecto de la progesterona exógena $\left(\mathrm{P}_{4}\right)$ sobre el desarrollo de la onda folicular fue estudiado en 25 hembras adultas, las cuales fueron distribuidas en 5 grupos: G0 (control); G1 (30 mg de $\mathrm{P}_{4} /$ día por 2 días); G2 (30 $\mathrm{mg} \mathrm{P}_{4} /$ día por 4 días); $\mathrm{G} 3$ (50 $\mathrm{mg} \mathrm{P}_{4} /$ día por 2 días) y $\mathrm{G} 4$ (50 $\mathrm{mg} \mathrm{P}_{4} /$ día por 4 días). El desarrollo de los folículos dominante $(\geq 7 \mathrm{~mm})$ y subordinados ( $>3 \mathrm{~mm}$ y $<7 \mathrm{~mm}$ ) fue monitoreado diariamente por ecografía transrectal de los ovarios, hasta el día 8 del inicio del tratamiento. El tamaño mínimo alcanzado por el folículo dominante en los grupos G1 (4.6 $\pm 0.9 \mathrm{~mm}), \mathrm{G} 3(5.1 \pm 1.5 \mathrm{~mm})$ y G4 $(5.3 \pm 1.6 \mathrm{~mm})$ fue menor $(\mathrm{P}<0.05)$ al grupo $\mathrm{G} 0(8.5 \pm 1.0 \mathrm{~mm})$. La regresión total del folículo dominante en los grupos G1 $(5.5 \pm 2.0 \mathrm{~mm}), \mathrm{G} 2(5.9 \pm 1.4 \mathrm{~mm})$ y G3 $(5.3 \pm 3.3 \mathrm{~mm})$ fue mayor $(\mathrm{P}<0.05)$ al grupo G0 $(0.9 \pm 1.4 \mathrm{~mm})$. El número de folículos subordinados tuvo un aumento significativo $(\mathrm{P}<0.05)$ en los grupos G1 y G4 entre los días 2 y 3 con respecto a los días 7 y 8 post inicio del tratamiento. Se demuestra que la progesterona exógena inhibe el desarrollo de los folículos dominantes y subordinados durante los días de tratamiento, y que luego de finalizado aumenta el número de folículos subordinados, probablemente debido a la regresión del folículo dominante que permite la emergencia de una nueva onda folicular.

Palabras clave: progesterona, ondas foliculares, folículos, llamas

\footnotetext{
1 Práctica privada

${ }^{2}$ Laboratorio de Reproducción Animal, FMV-UNMSM. E-mail: vleyvav@vet.unmsm.edu.pe

${ }^{3}$ Estación Experimental del Centro de Investigación IVITA-Maranganí, FMV-UNMSM
} 


\section{INTRODUCCIón}

La dinámica de la actividad folicular en llamas (Adams et al., 1990) y alpacas (Bravo y Sumar, 1985) se desarrolla a través de ondas foliculares, con la presencia de un folículo dominante. En animales con ciclo estrual definido, la actividad folicular es regulada por los niveles de progesterona secretada por el cuerpo lúteo (Leyva, 1996). Similar resultado se reporta en llamas gestantes y con fase luteal inducida, por el efecto inhibitorio de la progesterona en el desarrollo de los folículos dominante y subordinados (Adams et al., 1990).

En vacas se atribuye a la presencia de folículos dominantes la responsabilidad de la variabilidad entre animales en el intervalo ovulatorio (Ginther et al., 1989). De igual manera, se reporta una variabilidad en las tasas de ovulaciones múltiples en superovulación en llamas (Ratto et al., 1997) y alpacas en celo (Novoa et al., 1999; Velásquez, 1999), y alrededor del final de una fase luteal inducida (Correa et al., 1994; Bourke et al., 1995; Velásquez, 1999). Según la metodología de estos trabajos, el momento de la administración de las hormonas gonadotrópicas fue independiente del estadio de desarrollo del folículo dominante. Sin embargo, es probable que la respuesta a los tratamientos hormonales sea influenciada por las fases involucradas en el desarrollo del folículo dominante descritas en llamas (Adams et $a l ., 1990)$ y alpacas (Bravo y Sumar, 1985).

Últimamente se ha reportado el uso de progesterona exógena en dosis aparentemente fisiológicas en la inhibición del celo pre y post ovulatorio en alpacas (Leyva y García, 1999a) y en la sensibilización del eje hipotálamo-hipófisis-ovario en alpacas en celo, para incrementar la respuesta ovulatoria al estímulo coital del macho (Leyva y García, 1999b). Estos resultados sugieren que la progesterona exógena puede ser utilizada para regular la emergencia y desarrollo de los folículos dominantes y subordinados; por lo tanto, el objetivo del presente trabajo fue estudiar el efecto de la progesterona exógena sobre el desarrollo de la onda folicular en llamas.

\section{Materiales y Métodos}

\section{Ubicación}

El estudio se llevó a cabo durante los meses de marzo y abril del 2000 en el Fundo San Luis, perteneciente a la Estación Experimental IVITA-Maranganí de la Facultad de Medicina Veterinaria de la Universidad $\mathrm{Na}$ cional Mayor de San Marcos. El fundo se encuentra ubicado en el distrito de Nuñoa, provincia de Melgar, departamento de Puno, Perú, a una altitud aproximada de 4,200 msnm.

\section{Animales}

Se seleccionaron al azar 25 hembras con conducta de celo ante la presencia del macho y que presentaron un folículo preovulatorio $(\geq 7 \mathrm{~mm})$ al examen ecográfico.

\section{Diseño Experimental}

Se formaron los siguientes grupos de tratamientos $(n=5)$ en forma completamente al azar:

- G0: Grupo Testigo. No recibió ningún tratamiento

- G1: $30 \mathrm{mg}$ de progesterona/día, vía i.m., por 2 días consecutivos

- G2: $30 \mathrm{mg}$ de progesterona/día, vía i.m., por 4 días consecutivos

- G3: $50 \mathrm{mg}$ de progesterona/día, vía i.m., por 2 días consecutivos

- G4: $50 \mathrm{mg}$ de progesterona/día, vía i.m., por 4 días consecutivos

\section{Procedimiento Metodológico}

El desarrollo de los folículos dominantes ( $>7 \mathrm{~mm}$ ) y subordinados ( $>3 \mathrm{~mm}$ y $<7 \mathrm{~mm}$ ) 
fue monitoreado diariamente por ecografía transrectal de los ovarios, usando un ecógrafo B-mode Aloka 210 equipado con un transductor lineal tipo array de $5 \mathrm{MHz}$. Se consideró como día 0 al día de inicio del experimento. El experimento finalizó el día 8, cuando el $80 \%$ de las hembras retornaron en celo.

\section{Análisis de Datos}

El análisis estadístico fue realizado utilizando el SAS (Statistic Analisis System). La prueba de análisis de varianza (ANOVA) fue utilizada para analizar la diferencia por efecto de los tratamientos de los siguientes parámetros: (a) tamaño del folículo dominante en el día 0; (b) tamaño mínimo del folículo dominante; (c) grado de regresión folicular diario; y (d) regresión total del folículo dominante. Se utilizó la prueba de Tukey para establecer diferencias entre grupos. Además, el diámetro del folículo dominante y el número de folículos subordinados fueron analizados dentro de cada grupo durante el experimento, y día por día entre los 5 grupos, mediante ANOVA. Se utilizó la prueba de Tukey para evaluar diferencias entre promedios.

El coeficiente de correlación fue utilizado para establecer el grado de asociación entre el diámetro del folículo dominante y el número de folículos subordinados.

\section{Resultados}

\section{Tamaño del Folículo Dominante}

En la regresión de los folículos dominantes durante la fase experimental, el tamaño mínimo alcanzado en los animales de los grupos tratados con progesterona (G1, G3 y $\mathrm{G} 4)$ fue significativamente menor $(\mathrm{P}<0.05)$ que aquellos del grupo control (Cuadro 1), no obstante que el tamaño del folículo dominante al inicio del experimento (día 0 ) fue similar para los 5 grupos ( $\mathrm{P}>0.05$ ) (Cuadro 1). El grado de regresión folicular diaria desde el día 0 hasta el día en que se alcanza el tamaño mínimo (Cuadro 1) fue significativamente mayor $(\mathrm{P}<0.05)$ en los cuatro grupos tratados que en el grupo testigo. Asimismo, la regresión total del folículo dominante (Cuadro $1)$, fue significativamente mayor $(\mathrm{P}<0.05)$ en los grupos G1, G2 y G3 que en G0.

Al analizar la dinámica en el tamaño del folículo dominante dentro de cada grupo durante el experimento, se encontró una disminución significativa en $\mathrm{G} 1(\mathrm{P}<0.05)$ y G2 $(\mathrm{P}<0.01)$ (Cuadro 2).

Al comparar entre grupos, el folículo dominante del G0 a partir del día 3 comienza a hacerse significativamente mayor que los folículos dominantes de los demás grupos $(\mathrm{P}<$ 0.05 ), alcanzando el día 7 un tamaño significativamente mayor $(\mathrm{P}<0.01)$ que los folículos dominantes de G1, G2, G3 y G4 (Cuadro 2). El día 8 ya no se observan diferencias significativas $(P>0.05)$ entre los 5 grupos en relación al tamaño del folículo dominante.

\section{Emergencia de Folículos Subordinados}

Al analizar el número de folículos subordinados en cada grupo a través de los días, se observó una tendencia de incremento en los grupos tratados con progesterona (Fig. 1), siendo significativa $(\mathrm{P}<0.05)$ en el G1, entre los días $2(0.6 \pm 0.9)$ y $7(3.4 \pm 1.9)$; y en el G4 entre los días $3(1.0 \pm 1.2)$ y $8(3.8 \pm 1.9)$ después de iniciado el tratamiento (Cuadro 3 ).

\section{Relación Folículo Dominante y Folículos Subordinados}

Los coeficientes de correlación entre tamaño del folículo dominante y número de folículos subordinados para los grupos $0,1,2$, 3 y 4 fueron $-0.14,-0.37,-0.29,-0.28$ y 0.20 , respectivamente. Los coeficientes de correlación entre tamaño del folículo dominante y número de folículos subordinados para los días $0,1,2,3,4,5,7$ y 8 fueron $-0.28,-0.13$, 
Cuadro 1. Efecto de la progesterona sobre el folículo dominante en llamas (promedio \pm desviación estándar)

\begin{tabular}{lccccc}
\hline & $\mathrm{G} 0$ & $\mathrm{G} 1$ & $\mathrm{G} 2$ & $\mathrm{G} 3$ & $\mathrm{G} 4$ \\
\hline $\begin{array}{l}\text { Tamaño del folículo } \\
\text { dominante (d 0, mm) }\end{array}$ & $9.5 \pm 1.2$ & $10.1 \pm 2.5$ & $12.1 \pm 1.2$ & $10.4 \pm 4.1$ & $9.2 \pm 2.1$ \\
$\begin{array}{l}\text { Tamaño mínimo del } \\
\text { folículo dominante }(\mathrm{mm})\end{array}$ & $8.5 \pm 1.0^{\mathrm{a}}$ & $4.6 \pm 0.9^{\mathrm{b}}$ & $6.2 \pm 1.5^{\mathrm{ab}}$ & $5.1 \pm 1.3^{\mathrm{b}}$ & $5.3 \pm 1.6^{\mathrm{b}}$ \\
$\begin{array}{l}\text { Grado de regresión } \\
\text { folicular diario }(\mathrm{mm} / \mathrm{d})\end{array}$ & $0.2 \pm 0.2^{\mathrm{a}}$ & $1.3 \pm 0.4^{\mathrm{b}}$ & $0.9 \pm 0.4^{\mathrm{b}}$ & $0.9 \pm 0.4^{\mathrm{b}}$ & $1.1 \pm 0.3^{\mathrm{b}}$ \\
$\begin{array}{l}\text { Regresión total del } \\
\text { folículo dominante }(\mathrm{mm})\end{array}$ & $0.9 \pm 1.4^{\mathrm{a}}$ & $5.5 \pm 2.0^{\mathrm{b}}$ & $5.9 \pm 1.4^{\mathrm{b}}$ & $5.3 \pm 3.3^{\mathrm{b}}$ & $3.9+1.7^{\mathrm{ab}}$ \\
\hline
\end{tabular}

G0: Testigo; Gl y G2: $30 \mathrm{mg}$ de progesterona/día por 2 y 4 dias, respectivamente; G3 y G4: 50 mg de progesterona/día por 2 y 4 días, respectivamente

${ }^{1}$ Diferencia entre el día 0 y el día con el tamaño mínimo del folículo dominante

$a, b$ Letras diferentes dentro de filas indican diferencias significativas $(P<0.05)$

Cuadro 2. Efecto de la progesterona en la dinámica del tamaño del folículo dominante en llamas (promedio \pm desviación estándar)

\begin{tabular}{ccccccccc}
\hline \multirow{2}{*}{ Grupo } & \multicolumn{7}{c}{ Días post-inicio tratamiento } \\
\cline { 2 - 9 } & 0 & 1 & 2 & 3 & 4 & 5 & 6 & 7 \\
\hline G0 & $9.5 \pm 1.2$ & $10.6 \pm 1.1$ & $10.4 \pm 1.7$ & $10.8 \pm 2.5^{\mathrm{a}}$ & $11.7 \pm 4.1^{\mathrm{a}}$ & $11.2 \pm 4.8^{\mathrm{a}}$ & $12.0 \pm 4.7^{\mathrm{a}}$ & $11.7 \pm 5.1$ \\
G1 & $10.1 \pm 2.4^{\mathrm{x}}$ & $8.9 \pm 2.4^{\mathrm{xy}}$ & $8.4 \pm 2.1^{\mathrm{xy}}$ & $6.4 \pm 1.9^{\mathrm{bxy}}$ & $6.7 \pm 1.6^{\mathrm{bxy}}$ & $5.5 \pm 1.3^{\mathrm{by}}$ & $6.0 \pm 2.5^{\mathrm{bxy}}$ & $7.0 \pm 1.9^{\mathrm{xy}}$ \\
G2 & $12.1 \pm 1.2^{\mathrm{x}}$ & $11.4 \pm 1.1^{\mathrm{xy}}$ & $10.6 \pm 0.8^{\mathrm{xy}}$ & $8.9 \pm 1.2^{\mathrm{abyz}}$ & $8.4 \pm 1.4^{\mathrm{abzw}}$ & $7.3 \pm 1.8^{\mathrm{abw}}$ & $7.1 \pm 1.5^{\mathrm{bw}}$ & $7.4 \pm 1.3^{\mathrm{w}}$ \\
G3 & $10.4 \pm 4.1$ & $9.6 \pm 4.7$ & $7.8 \pm 2.4$ & $7.4 \pm 2.2^{\mathrm{ab}}$ & $6.3 \pm 2.4^{\mathrm{b}}$ & $5.6 \pm 2.3^{\mathrm{b}}$ & $6.2 \pm 0.9^{\mathrm{b}}$ & $6.9 \pm 1.3$ \\
G4 & $9.2 \pm 2.1$ & $8.0 \pm 2.4$ & $7.7 \pm 2.8$ & $6.8 \pm 2.7^{\mathrm{b}}$ & $6.4 \pm 2.1 \mathrm{~b}^{\mathrm{b}}$ & $6.3 \pm 2.1^{\mathrm{b}}$ & $5.9 \pm 0.9 \mathrm{~b}$ & $7.4 \pm 2.2$ \\
\hline
\end{tabular}

G0: Testigo; Gl y G2: $30 \mathrm{mg}$ de progesterona/día por 2 y 4 días, respectivamente; G3 y G4: 50 mg de progesterona/día por 2 y 4 días, respectivamente

$a, b$ Letras diferentes dentro de columnas indican diferencias significativas $(P<0.05)$

$x, y, z, w$ Letras diferentes dentro de filas indican diferencias significativas $(P<0.05)$

$0.31,0.12,-0.08,-0.18,-0.43$ y -0.28 , respectivamente. La relación entre el tamaño del folículo dominante y el número de folículos subordinados puede apreciarse en la Fig. 1.

\section{Discusios}

El presente trabajo es el primer reporte que demuestra que la administración de progesterona exógena altera el desarrollo de la onda folicular en llamas, ocasionando una disminución del tamaño del folículo dominante a través de un mayor grado de regresión, una disminución del número de folículos subordinados durante el periodo de tratamiento con progesterona, y posteriormente un incremento de estos folículos subordinados al desaparecer el efecto de la progesterona.

Similar efecto en la disminución del tamaño del folículo dominante por administra- 

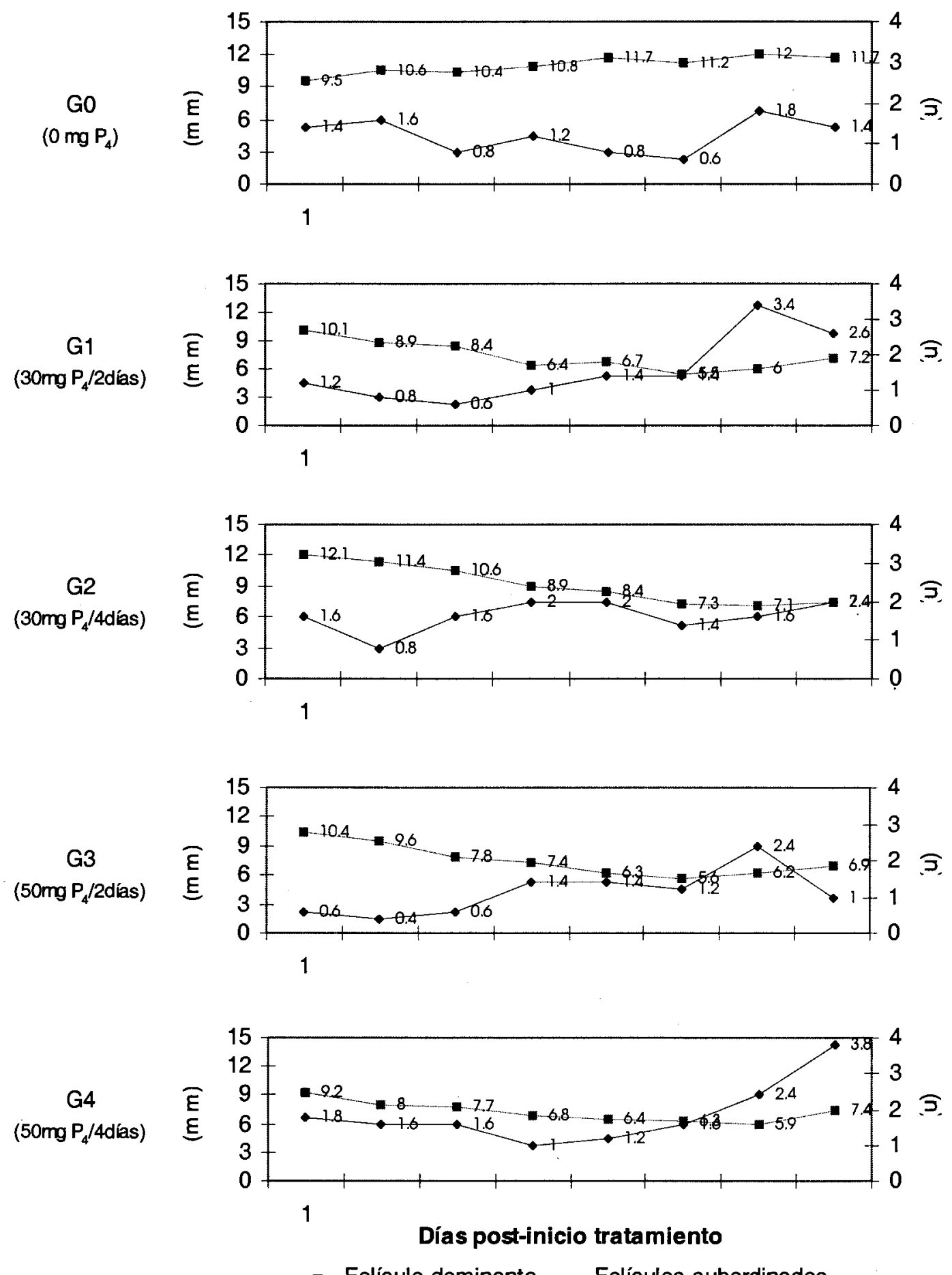

Figura 1. Efecto de la progesterona sobre el tamaño del folículo dominante y número de folículos subordinados 
Cuadro 3. Efecto de la progesterona sobre el número de folículos subordinados en llamas (promedio \pm desviación estándar)

\begin{tabular}{ccccccccc}
\hline Grupo & \multicolumn{7}{c}{ Días post-inicio tratamiento } \\
\cline { 2 - 8 } & 0 & 1 & 2 & 3 & 4 & 5 & 6 & 7 \\
\hline $\mathrm{G} 0$ & $1.4 \pm 0.9$ & $1.6 \pm 1.5$ & $1.0 \pm 0.8$ & $1.2 \pm 0.8$ & $0.8 \pm 0.8$ & $0.8 \pm 0.5$ & $1.6 \pm 1.3$ & $1.4 \pm 1.5$ \\
$\mathrm{G} 1$ & $1.2 \pm 0.8^{\mathrm{ab}}$ & $0.8 \pm 1.1^{\mathrm{ab}}$ & $0.6 \pm 0.9^{\mathrm{a}}$ & $1.0 \pm 1.0^{\mathrm{ab}}$ & $1.4 \pm 1.4^{\mathrm{ab}}$ & $1.4 \pm 1.3^{\mathrm{ab}}$ & $3.4 \pm 1.9^{\mathrm{b}}$ & $2.6 \pm 1.7^{\mathrm{ab}}$ \\
$\mathrm{G} 2$ & $1.6 \pm 1.9$ & $0.8 \pm 1.3$ & $1.6 \pm 1.5$ & $2.0 \pm 2.0$ & $1.8 \pm 1.6$ & $1.4 \pm 0.9$ & $1.6 \pm 0.9$ & $2.0 \pm 1.4$ \\
$\mathrm{G} 3$ & $0.6 \pm 0.9$ & $0.4 \pm 0.9$ & $0.6 \pm 1.3$ & $1.4 . \pm 1.3$ & $1.4 \pm 1.1$ & $1.2 \pm 1.3$ & $2.6 \pm 1.9$ & $1.0 \pm 0.8$ \\
$\mathrm{G} 4$ & $1.8 \pm 1.3^{\mathrm{ab}}$ & $1.6 \pm 0.5^{\mathrm{ab}}$ & $1.6 \pm 1.3^{\mathrm{ab}}$ & $1.0 \pm 1.2^{\mathrm{a}}$ & $1.2 \pm 1.3^{\mathrm{ab}}$ & $1.6 \pm 1.1^{\mathrm{ab}}$ & $2.4 \pm 1.5^{\mathrm{ab}}$ & $3.8+1.9^{\mathrm{b}}$ \\
\hline
\end{tabular}

G0: Testigo; G1 y G2: $30 \mathrm{mg}$ de progesterona/día por 2 y 4 días, respectivamente; G3 y G4: $50 \mathrm{mg}$ de progesterona/día por 2 y 4 días, respectivamente

$a, b$ Letras diferentes dentro de filas indican diferencias significativas $(P<0.05)$

ción de progesterona exógena es reportado por Leyva (1996) en ovinos y por Adams et al. (1992) en bovinos. Este efecto también es comparable al menor tamaño del folículo dominante expuesto a niveles altos de progesterona en la fase luteal inducida en llamas (Adams et al., 1990) y en la fase luteal del ciclo estral en ovinos (Leyva, 1996) y bovinos (Ginther et al., 1989). Es probable que este efecto fuera mediado por interferencia en el desarrollo del folículodominante debidoal efecto inhibitorio de la progesterona exógena en la secreción de la GnRH hipotalámica y, consecuentemente, en la secreción de la LH hipofisiaria (Stevenson, 1997).

Hubo disminución en el número de folículos subordinados durante los días de tratamiento con progesterona; sin embargo, después de finalizado el tratamiento, esta situación fue revertida. Estos resultados sugieren que la progesterona exógena ejerce un efecto inhibitorio en el desarrollo de los folículos subordinados. Este efecto es comparable a la disminución del número de folículos pequeños en ovejas en anestro, tratadas con progesterona exógena (Leyva, 1996). Se ha demostrado que la progesterona inhibe la secreción de LH y en menor grado la FSH (Savio et al., 1993, citado por Adams, 1994), las cuales son necesarias para el crecimiento folicular; sin embargo, el efecto inhibitorio del folículo dominante en los subordinados ha sido atribuido a reguladores locales ováricos como la inhibina que controla la secreción de FSH (Fortune, 1994).

En condiciones fisiológicas normales, el desarrollo de los folículos subordinados es generalmente suprimido cuando el folículo dominante se encuentra en su fase estática, con una relación inversamente proporcional entre el número de folículos detectados y el tamaño del folículo dominante $(r=-0.2)$ (Adams et al., 1990). Esto concuerda con la relación de - 0.14 encontrado en el grupo control ( $\mathrm{G} 0$ ), mientras que en los grupos tratados con progesterona la relación se vuelve positiva en los días $2(\mathrm{r}=0.31)$ y $3(\mathrm{r}=0.12)$, es decir, que disminuye tanto el número de folículos subordinados como el tamaño del folículo dominante, para luego volverse negativa, estableciéndose una asociación fuerte en el día $7(r=-0.43)$. Estos resultados sugieren que al inicio del tratamiento la progesterona ejerce un efecto inhibitorio tanto en el folículo dominante como en los subordinados, y que luego el aumento del número de folículos subordinados sería el resultado de la continua regresión del folículo do- 
minante, y la desaparición del efecto inhibitorio de la progesterona después de suspenderse el tratamiento.

En vaquillas, Adams (1994) refiere que el mayor número de folículos en tratamientos superovulatorios se consigue cuando el tratamiento se inicia el día de la emergencia de la onda folicular. En condiciones fisiológicas normales, después de la fase estática ocurre la regresión del folículo dominante. Por consiguiente, en las hembras tratadas con progesterona se ha reproducido este efecto para favorecer la emergencia de una nueva onda folicular y ello parece haber ocurrido entre los días 3 y 4 luego de iniciado el tratamiento con progesterona, donde se observa el iniciodel incremento del númerode folículos subordinados (Fig. 1). Esta información es importante para un uso más eficiente de las técnicas de superovulación en llamas y sería conveniente identificar el mejor momento para iniciar un tratamiento superovulatorio en relación al momento de la emergencia de la nueva onda folicular.

\section{Conclusiones}

La progesterona exógena inhibe el desarrollo de los folículos dominante y subordinados en llamas, y al finalizar el tratamiento con progesterona, aumenta el número de folículos subordinados. Además, la progesterona exógena inhibe la receptividad sexual desde el día 2 de iniciado el tratamiento hasta los días 7 a 8.

\section{Literatura Cimada}

1. Adams, G.P.; J. Sumar; O. Ginther. 1990. Effects of lactational and reproductive status on ovarian follicular waves in llamas (Lama glama). J. Reprod. Fert. 90: 535-545.

2. Adams, G.P.; R. Matteri; O. Ginther. 1992. Effect of progesterone on ovarian follicles, emergence of follicular waves and circulating follicle-stimulating hormone in heifers. J. Reprod. Fertil. 96: 627-640.

3. Adams, G.P. 1994. Control of ovarian follicular wave dynamics in cattle: implications for synchronization and superstimulation. Theriogenology 41: 19-24.

4. Bourke, D.A.; C. Kyle; T. McEvoy; P. Young; C. Adam. 1995. Superovulatory responses to eCG in llamas (Lama glama). Theriogenology 44: 255-268.

5. Bravo, P.W.; J. Sumar. 1985. Actividad folicular del ovario en la alpaca. Res. $5^{\mathbf{a}}$. Convención Internacional sobre Camélidos Sudamericanos. Cusco. p 7.

6. Correa, J.E.; M. Ratto; R. Gatica. 1994. Actividad estral y respuesta ovárica en alpacas y llamas tratadas con progesterona y gonadotropinas. Arch. Med. Vet. 26: 59-64.

7. Fortune, J.E. 1994. Ovarian follicular growth and development in mammals. Biol. Reprod. 50: 225-232.

8. Ginther, O.J.; L. Knopf; J. Kastelic. 1989. Temporal associations among ovarian events in catttle during oestrus cycles with two and three follicular waves. J. Reprod. Fertil. 87: 223-230.

9. Leyva, V. 1996. Follicular activity and ovulation of ewes during the breeding season and anestrus. Tesis PhD. Univ. Guelph, Canadá. 278 p.

10. Leyva, V.; W. García. 1999a. Efecto de la progesterona exógena sobre la función del cuerpo lúteo de alpacas. Res. II Cong. Mund. Camélidos. Cusco. p 87.

11. Leyva, V.; W. García. 1999b. Efecto de la progesterona exógena y endógena en alpacas en celo sobre la ovulación, fertilización y gestación. Res. II Cong. Mund. Camélidos. Cusco. p 89.

12. Novoa, C.; E. Franco; W. García; D. Pezo. 1999. Dosis de gonadotropinas (eCG y hCG), superovulación y obtención de embriones en alpacas. Rev. Inv. Vet. Perú 10: 48-53.

13. Ratto, M.H.; R. Gatica; J. Correa. 1997. Timing of mating and ovarian response in llamas (Lama glama) treated with pFSH. Anim. Reprod. Sci. 48: 325-330. 
14. Stevenson, J.S. 1997. Clinical reproductive phisiology of the cow. En: Current therapy in large animal. Theriogenology, Vol. I, Cap. 32. Youngquist, R.S. (ed). Ed. W.B. Sauders Company, Philadelphia.
15. Velásquez, C. 1999. Tratamiento superovulatorio con PMSG aplicado en fase folicular versus fase progestacional en alpacas. Tesis Maestría. Fac. Med. Vet., Univ. Nac. Mayor San Marcos. Lima. $70 \mathrm{p}$. 